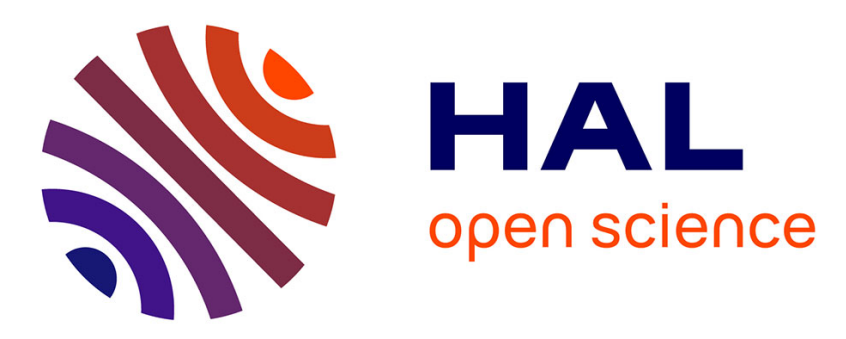

\title{
Mobility of Humanoid Robots: Stepping over Large Obstacles Dynamically
}

Björn Verrelst, Kazuhito Yokoi, Olivier Stasse, Hitoshi Arisumi, Bram

Vanderborght

\section{- To cite this version:}

Björn Verrelst, Kazuhito Yokoi, Olivier Stasse, Hitoshi Arisumi, Bram Vanderborght. Mobility of Humanoid Robots: Stepping over Large Obstacles Dynamically. IEEE International Conference on Mechatronics and Automation, Jun 2006, Henan, China. pp.1072 - 1079, 10.1109/ICMA.2006.257774 . hal-01117866

\section{HAL Id: hal-01117866 \\ https://hal.science/hal-01117866}

Submitted on 18 Feb 2015

HAL is a multi-disciplinary open access archive for the deposit and dissemination of scientific research documents, whether they are published or not. The documents may come from teaching and research institutions in France or abroad, or from public or private research centers.
L'archive ouverte pluridisciplinaire HAL, est destinée au dépôt et à la diffusion de documents scientifiques de niveau recherche, publiés ou non, émanant des établissements d'enseignement et de recherche français ou étrangers, des laboratoires publics ou privés. 


\section{Mobility of Humanoid Robots: Stepping over Large Obstacles Dynamically}

\author{
Björn Verrelst, Kazuhito Yokoi, Olivier Stasse, and Hitoshi Arisumi \\ Joint Japanese-French Robotics Laboratory (JRL) \\ National Institute of Advanced Industrial Science and Technology (AIST) \\ AIST Central 2, 1-1-1 Umezono, Tsukuba, Ibaraki, Japan 305-8568 \\ \{bjorn.verrelst,kazuhito.yokoi,olivier.stasse,h-arisumi\}@aist.go.jp
}

\author{
Bram Vanderborght \\ Robotics \& Multibody Mechanics Research Group \\ Vrije Universiteit Brussel \\ Pleinlaan 2, 1050 Brussel, Belgium \\ bvdborgh@vub.ac.be
}

\begin{abstract}
Humanoid robots are getting increasingly attention in the robotics community, not only for the scientific challenge of the complex multibody system issues and mechatronic designs, but also due to their high mobility and versatility. Humanoid robots have the potential to navigate through complex environments such as the standard living surrounding of humans. This is mainly due to the bipedal legged nature of the robotic system, which allows higher mobility than its wheeled counterpart. One of the advantages is that it can negotiate obstacles by stepping over them, which is the topic of the work presented in this paper. The main focus of this research is to investigate stepping over large obstacles. Previous work has reported on algorithms using quasi-static balancing, which resulted in somehow unnatural slow motions. This work however is focussing on stepping over larger obstacles in a fluent dynamic motion, using stability criteria on zero moment point instead of center of gravity. All the work is formulated in function of the elaborate HRP-2 humanoid research platform. In this paper a preliminary 2D study on stepping over leg trajectories and their dynamic implications on the overall stability are investigated. The paper discusses the implementation of the stepping over procedure in the overall dynamic motion generator, the implications on the kinematics and dynamics and finally the actual stepping over foot trajectory planner.
\end{abstract}

\section{INTRODUCTION}

The last two decades, substantial progress has been made in the field of legged robots. One of the important and astonishing events happened in Tokyo on December 20th in 1996 when Honda Motor Corporation presented there humanoid "Honda Humanoid Robot" [1] after ten years of secret research. This event seems to have triggered a robot technology race between the big Japanese companies to develop their own legged walking machine. As such the idea of humanoid robots leaves science fiction and is becoming more and more realistic. The latest developments, worldwide, show elaborate and nice hardware models featuring fully equipped humanoids. In the past, the main research effort consisted in hardware design and walking pattern generators, where as recently research centers begin to focus on more specific topics in increasing the autonomy and skills of humanoids. After all, a humanoid robot might be the future assistant to humans in the latter's own environment due to its specific layout. A lot of research is going on in a broad field of expertise, e.g. vision guided self localization [2], [3], humanoid cognitive architectures [4] and task-oriented whole body control [5], [6]. Another important topic, specifically related to humanoids, is the autonomy towards navigation in a complex human environment. In this frame work a lot of attention goes to path planning, focussing on obstacle avoidance and goal seeking [7], [8]. These studies do incorporate the specific abilities of humanoids towards mobility, which is in fact the use of discrete footholds and as such being able to step over obstacles. But with respect to the latter, the regarded obstacles generally are small, while actually a humanoid has the capability of negotiating larger obstacles which are often encountered in a standard human environment. Of course this requires specific strategies, which is the topic of this paper. The presented work specifically focuses on implementation on the elaborate humanoid research platform HRP-2 [9].

Previous work on stepping over large obstacles, conducted by Guan [10], [11], investigated the feasibility of the stepping over, determining the maximum dimension of the obstacle which can be negotiated, and proposed a stepping over trajectory planning. Hereby focusing on quasi-static stepping over procedures by keeping the projection of the global Center of Gravity $(C o G)$ of the robot within the polygone of support. Since the postural stability only takes into account the $C o G$, the motion of the robot has to be slow in order not to induce substantial accelerations and as such not demanding for dynamic stability criteria, e.g. Zero Moment Point $(Z M P)$ [12]. If large obstacles are considered, this quasi-static stepping over motion has a quite unnatural resemblance due to the continuous restricting balancing of the CoG. Moreover, a large double support phase is required, in order to shift the $C o G$ from the rear to the front during the particular phase. This implies restrictions regarding geometric dimensions of the obstacles which can be negotiated.

On the contrary, a dynamic stepping over procedure cancels the restriction of the $C o G$ balancing and allows for shorter double support phases. A dynamic walking pattern is characterized by postural stability on the $Z M P$ criterion and allows the $C o G$ to leave the supporting foot as long as the $Z M P$ stays within the polygon of support. Of course, for the stepping over procedure this allows for more freedom on the specific upper-body postures of the robot, since the $Z M P$ is not only determines by positions, but also by accelerations of the $C o G$. Moreover, the $C o G$ can be shifted from one side of the obstacle 
to the other during a single support phase, which in theory should allow for only using an instantaneous double support phase, if running is not regarded. This results in larger obstacle dimensions which can be negotiated.

Since the stability criterion is not quasi-static anymore, the stepping over can be done in one fluent and natural motion, but of course this demands for more complex motion planning. In fact the stepping over procedure is a "go and no return" approach which requires careful obstacle stepping over planning. Moreover, a robust dynamic pattern generator is needed which can cope with the dynamical effects of large swing leg retractions and alike.

This paper discusses the kinematic and dynamic implications of dynamic stepping over large obstacles and proposes a preliminary foot trajectory planner in the sagittal plane. A specific dynamic pattern generator currently incorporated in the HRP-2 robot is used, and the dynamic implications on the performance of the pattern generation are simulated with a two dimensional 7-link lumped mass model.

\section{Overall Dynamic Pattern Generator b y PREVIEW CONTROL}

As mentioned in the previous section, a robust dynamic pattern generator is required. The last decade a lot of research work has been done on the development of online stable dynamic walking pattern generators. The pattern generator used in this paper is based on the Three Dimensional Linear Inverted Pendulum Mode (3D-LIPM) [13] which uses a simplified single mass inverted pendulum model representing the center of mass of the humanoid robot pivoting around the ZMP point. Additionally, the mass is constraint to move on a parametric surface. One solution of this simplified model applied as a pattern generator solves the discretized equations of motion by optimal preview control. Since this method and its features are extensively used for the stepping over procedure, a short description is given here. Further detailed information can be found in the work of Kajita [14]. For

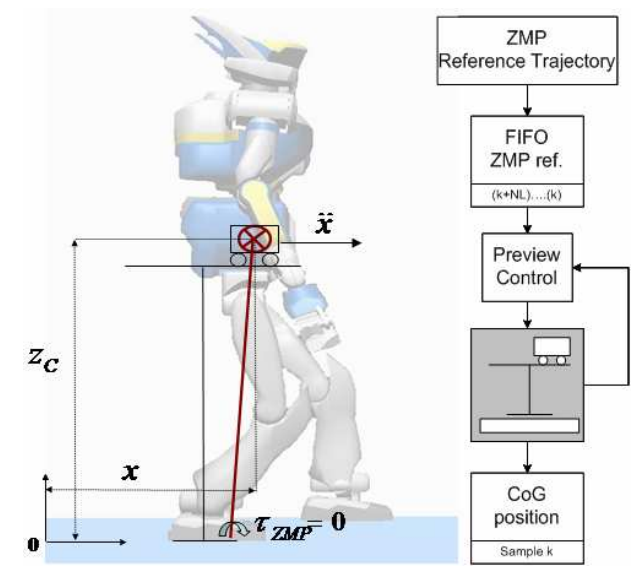

Fig. 1. The cart-table model with preview control scheme

regular walking, the specific constraint surface is a horizontal plane at a constant height, for which the basic inverted pendulum equations are easily explained with the cart-table model of the robot motion. The motion of the $C o G$ of the robot is that of a cart moving on a horizontally positioned pedestal table with negligible mass, as depicted in Fig. 1, hereby using two cart-table representations for the horizontal motion in the sagittal (x-z) and the frontal (y-z) plane separately. The basic equations for this simplified representation, linking the $Z M P$ to the $C o G$, are as follows [14]:

$$
\begin{aligned}
\ddot{x} & =\frac{g}{z_{c}}\left(x-p_{x}\right) \\
\ddot{y} & =\frac{g}{z_{c}}\left(y-p_{y}\right)
\end{aligned}
$$

With $x, y$ the moving coordinates of the $C o G, z_{c}$ the constant height of the $C o G$, and $p_{x}, p_{y}$ the position of the ZMP. If the cart is positioned near the edge of the table, the latter tends to tilt due to the small supporting area (comparable with a robot foot). But with proper accelerations, the ZMP can still be within the supporting area and as such the table will keep upright. This corresponds to the basic idea of dynamic walking of a humanoid, and is also of great importance for the dynamic stepping over. The $C o G$ of the robot might already be over the obstacle but the robot can still be supported by the foot in front of the obstacle, which is not possible with a quasi-static motion.

The main idea of the pattern generator is to plan the motion of the $C o G$, approximated by the hip motion, in function of desired ZMP trajectories determined by the foothold sequences. The problem is regarded as a $Z M P$ servo control implementation, trying to track the $Z M P$ by servo control of the horizontal acceleration. The use of preview control for solving the Eqs. (1) requires future information of the desired $Z M P$ (foothold) planning as is explained by the scheme in Fig. 1. For each sample $k$ with sample time $T, N L$ future $Z M P$ input points are stored in a fifo (first in first out) buffer and all this information contributes to the calculation of the actual position of the $C o G$. An important parameter influencing the result is the height of the $\operatorname{CoG}\left(Z_{c}\right)$ which is supposed to be constant according to the cart-table model.

\section{DYNAMic IMPLiCATIONS OF StePPING OVER PROCEDURE}

The preliminary qualitative study in this paper only focuses on the motion in the sagittal plane, since the essential difficulties are related to the sagittal motion. The dynamic stability in this paper is represented by ZMP calculations based on a seven link model of HRP-2 in which all parts above the hip, including head and arms, are integrated as one upper-body, since obstacle stepping over mainly involves the lower parts of the robot.

In the previous work on quasi-static stepping over the planning of the leg motions was done from bottom to top, meaning that first the foot trajectories were selected and consequently corresponding hip motions. In this study, on the contrary, the basic motion to start with is the hip motion generated by the dynamic pattern generator to ensure overall dynamic stability. 
Subsequently, the kinematics of the leg motions are planned in function of this corresponding hip motion. In this context the following issues are important:

- Dynamical effects disturbing the ZMP placement due to large leg swing motions.

- Dynamical effects of changing hip heights during the step over procedure.

In general, humanoid robots tend to walk with bend knees, thus at low hip heights, to avoid singular configurations at knee stretching. Of course, a procedure to step over large obstacle demands for an increased hip height, while in fact the pattern generator, as described in the previous section, considers only hip motions at constant height. Changing this height will of course influence the dynamic performance of the pattern generator. The same counts for large leg swing motions, since the formulation of the previous section only takes into account the global $C o G$ and not the separate motions of the limbs. With respect to this issue, Kajita [14] proposes a re-feeding of the complete multibody calculated $Z M P$ trajectory into the preview control by means of taking the error between the latter and the desired $Z M P$ trajectory. This error $(\triangle Z M P)$ is again presented as input of a second stage of preview control with the same cart-table model, resulting in deviations of the horizontal motion of the $\operatorname{CoG}(\Delta \mathrm{CoG})$. The complete scheme of this implementation is given in Fig. 2, which also includes the stepping over planner, elaborated on in the next section. Thus, the complete control loop needs $2 N L$ future $Z M P$ input points: $N L \triangle Z M P$ calculations are required to determine the final robot configuration at sample point $k$, but each of these $N L \triangle Z M P$ values are respectively derived from a calculated $C o G$ value during the first preview round, which in turn needs $N L Z M P$ input points.

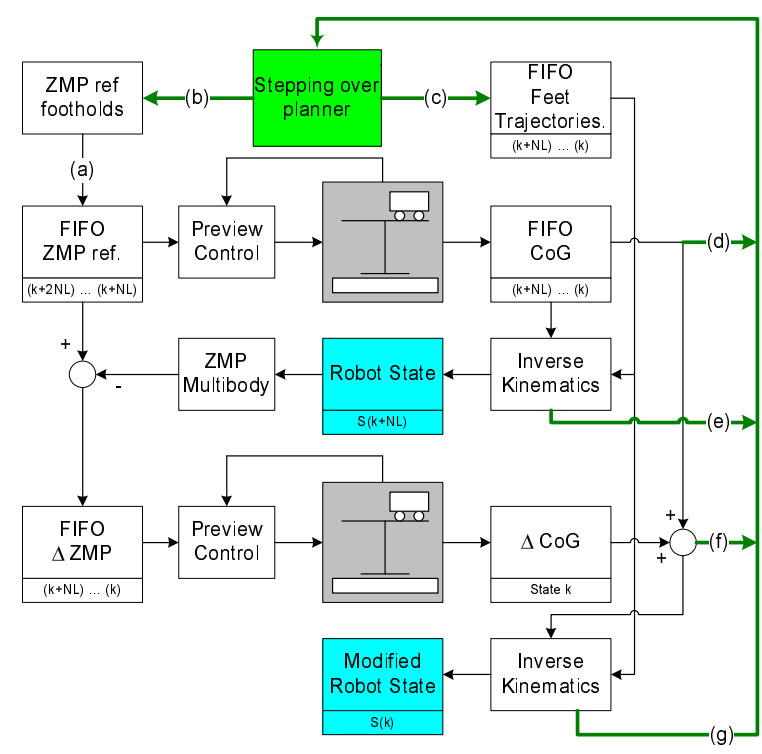

Fig. 2. Global preview control scheme

The main point is that the impact of the deviations between the multibody model and the inverted pendulum model are cancelled by the second preview loop. And for the case of stepping over large obstacles the same principle can be applied. Both leg swing motions and change in hip height during walking can be seen as deviations with respect to the base cart-table model. As long as the deviations are not too severe, the second preview loop can serve as a correcting unit. Note that it is essential for the inertial parameters of the upper-body (including arms and head) to be much larger than those of the leg, otherwise this second preview loop will not be sufficient. The same counts for the planning of the large leg swing motions. One can not implement random trajectories, since large accelerations will have a negative effect on the performance of the second correcting preview loop and eventually make it fail towards dynamic stability.

Fig. 3 shows the intended desired foothold sequence by means of the ZMP course. The graphs depict the $Z M P$ and $C o G$ plots, both after the $1^{\text {st }}$ and $2^{\text {nd }}$ preview loop. The first graph gives the complete time course and in the second graph a detail is given on one specific step. The simulation creating these graphs considers a stepping over of an obstacle with height of $20 \mathrm{~cm}$ and width of $5 \mathrm{~cm}$. In a first step the hip height is increased to the necessary hip height required during double support, in the subsequent step the first leg is going over the obstacle followed by an almost instantaneous double support phase with the obstacle positioned in between the legs. Next, the second leg retracts and positions behind the obstacle while an additional variation of the hip height is applied. And during the last step the hip lowers back to normal walking height. The specific planning of all these motions is explained in the next section. It is clear that both large leg swing and hip height variation have a substantial effect on the actual $Z M P$ position in the first preview loop, but that the second preview loop is able to cancel these disturbing effects. Of course, a careful planning of the leg swing motions is required.

\section{Foot Trajectory Planning}

\section{A. Feasibility during Double Support}

Using a dynamic stepping over procedure has the important advantage of a short double support phase, contrary to a quasi static procedure since the $C o G$ can be behind the obstacle while the supporting foot is still in front of it. This leads to a kinematical advantage concerning the feasible dimensions of the obstacle which can be negotiated.

The actual leg layout of HRP-2 and the closed kinematic chain during the double support phase makes this phase mainly determine the actual obstacles which can be stepped over. As such the stepping over planner starts here and calculates the step length, step height and foothold positions during the stepping over procedure. Fig. 4 shows all the essential parameters which are of concern for these calculations. The obstacle is regarded to be rectangular with certain width $O_{w}$ and height $O_{h}$. For the stepping over trajectory planning a safety margin $\left(S_{w}, S_{h}\right)$ around the obstacle is included, not only to cope with deviations of calculated kinematics during the actual stepping over, but mainly regarding the uncertainty 

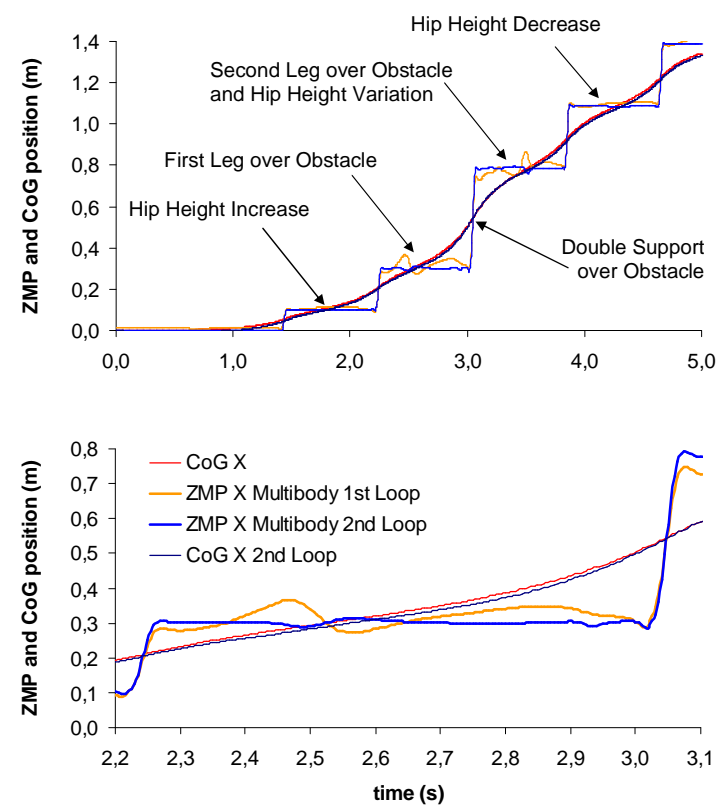

Fig. 3. ZMP and CoG position during walking including stepping over of obstacle

of the vision system, determining the obstacle dimensions, which will be implemented in the future.

The feasibility study is a kinematical study which calculates a collision free configuration with minimal step-length $\left(X_{a_{D S}}\right)$ and minimal hip height $\left(Z_{h_{D S}}\right)$, for large obstacles. For small obstacles the standard walking step-length and hip height are selected. The minimal values are selected in order to be as far as possible out of range of a singular leg overstretch configuration. For the same reason a minimum angle $\left(q_{\min }\right)$ for the knee angle $\left(q_{k}\right)$ is considered during the step-length determination.

As mentioned, the leg layout substantially limits the available space for the obstacle. And the determination of the possible configurations in this double support phase relies on collision detection between both lower legs and the obstacle. These calculations use a simplified representation of the leg layout, considering several line segments: $\left(l_{1}, l_{2}\right) . .\left(l_{3}, l_{4}\right)$ for the front side of the lower leg, and $\left(l_{5}, l_{6}\right),\left(l_{6}, l_{7}\right)$ for the rear side. Respective collision detections between all the line segments formed by $l_{1}$ to $l_{4}$ with the obstacle line segments formed by the points $o_{1}$ to $o_{3}$ are performed for the leg standing in front of the obstacle. And the same is done for the leg standing behind the obstacle for the line segments $l_{5}$ to $l_{7}$ with respect to obstacle line segments $o_{2}$ to $o_{4}$. Detection of a collision is performed by calculation of intersection between two line segments which is straightforward by considering an oriented area (e.g. $A_{l_{1}, l_{2}, o_{1}}$ ) formed by respectively three end points involved [10]:

$$
\begin{aligned}
& A_{l_{1}, l_{2}, o_{1}}= \\
& \quad x_{l_{1}}\left(z_{l_{2}}-z_{o_{1}}\right)+x_{l_{2}}\left(z_{o_{1}}-z_{l_{1}}\right)+x_{o_{1}}\left(z_{l_{1}}-z_{l_{2}}\right)
\end{aligned}
$$

No intersection occurs between line segment $\left(l_{1}, l_{2}\right)$ and

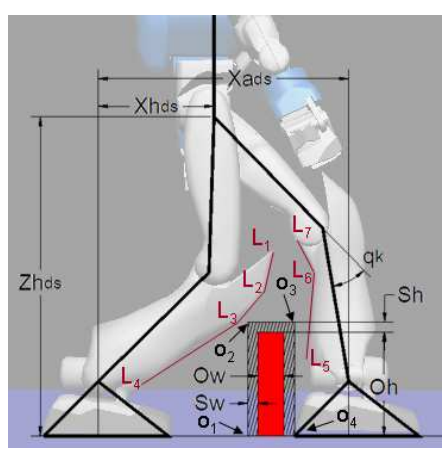

Fig. 4. Double support phase feasibility

$\left(o_{1}, o_{2}\right)$ if:

$$
\max \left(A_{l_{1}, l_{2}, o_{1}} A_{l_{1}, l_{2}, o_{2}}, A_{o_{1}, o_{2}, l_{1}} A_{o_{1}, o_{2}, l_{2}}\right)>0
$$

Since the rear leg is most likely to collide with the obstacle, due to the knee which is directed towards the obstacle, the heel of the front foot behind the obstacle is positioned near the safety boundary around the obstacle at point $o_{4}$. Thus, once the step-length is determined, both foothold positions of front and rear foot are fixed. Now there is one important parameter which resides in the calculations of the feasible step-length. The kinematical calculation of the closed loop formed by the two legs involves the horizontal hip distance $\left(X_{h_{D S}}\right)$, which in fact is determined by the dynamic pattern generator. But the pattern generator on the contrary first needs the input of $Z M P$ trajectories and foothold positions. Therefor the calculation of the step-length $\left(X_{a_{D S}}\right)$, and consequently foothold position, by the stepping over planner uses a parameter $\left(\delta_{D S}\right)$ which determines the position of the hip during double support in function of the step-length:

$$
X h_{D S}=\delta_{D S} X_{a_{D S}}
$$

The value of this parameter originates from a previously stored table containing an estimate for different step-lengths created by the pattern generator, for which a specific step-time (here $0.8 \mathrm{~s}$ ) has to be chosen. As it turns out this $\delta_{D S}$ value does not vary too much after application of the second preview control. This can be witnessed in the first graph of Fig. 3, where the intersection of both $C o G$ courses, before and after second preview, with the vertical line of the $Z M P$ does not vary substantially.

In summary, as a result of the feasibility calculation, first of all can be decided if a specific obstacle can be negotiated by stepping over it or not. And in case this decision is positive, the footholds and consequently the desired ZMP course can be determined. This is indicated by the flow line $(b)$ in the general scheme of Fig. 2. The next step is to plan the ankle trajectory during the step over procedure.

\section{B. Polynomial Ankle and Hip Height Trajectory Planner}

For both feet, to be positioned behind the obstacle, a separate polynomial planning of the ankle trajectories is used. 
But the stepping over of the last foot is the most awkward one, due to the bend knee configuration directed downwards. As such the explanation of the planning algorithm in this paper is focussed on the second foot only. Specific polynomial trajectories are designed for the ankle point, relative foot angle and additionally hip height motion. These trajectories form the base motion which are subsequently adapted to induce collision free motion. With respect to dynamic stability and the two preview loops, the polynomial planning is also developed in two stages.

The motion for the ankle trajectory is established with two polynomials (with respect to time), for the horizontal and vertical displacement of the ankle point separately. These polynomials take into account the start and end conditions of the foot, which commences in the double support phase calculated in the previous section, in front of the obstacle, and ends behind the obstacle just before the next normal double support phase. Thus the conditions on hip height and foot position, already established during the feasibility, are consequently taken into account here. Besides initial conditions in the end positions, additionally two intermediate ankle positions $\left(P_{1}\right.$ and $\left.P_{2}\right)$ are included, as depicted in picture (a) and (d) of Fig. 5. Ankle position $P_{1}$ is set at intermediate local time $t_{1}$ (chosen) such that the knee does not collide with the obstacle while the foot is still in front of the obstacle. In fact knee angle $q_{k}$ is chosen and consequently the hip angle $q_{h}$ is determined, as such that the knee does not interfere with the safety boundary of the obstacle and that it is in the neighborhood of point $O_{2}$. This provides a first safe (collision free) intermediate ankle position. The next position is determined at intermediate time $t_{2}$ (chosen) for the foot being already behind the obstacle. Here the ankle coordinates are selected such that the foot does not interfere with safety zone of the obstacle, after choosing a relative intermediate angle $q_{a}$. For the orientation of the foot the same foot angle choice is made at intermediate time $t_{1}$. Consequently, three $7^{\text {th }}$ order polynomials are establish, two for the $X$ and $Z$ ankle coordinate and a third for the relative ankle orientation $q_{a}$ :

$$
\begin{aligned}
X_{a}(t) & =\sum_{i=0}^{7} a_{i}^{X_{a}} t^{i} \quad ; \quad Z_{a}(t)=\sum_{i=0}^{7} a_{i}^{Z_{a}} t^{i} \\
q_{a}(t) & =\sum_{i=0}^{7} a_{i}^{q_{a}} t^{i}
\end{aligned}
$$

By using $7^{\text {th }}$ order polynomials, both speed and acceleration conditions at the end points can be taken into account to perform a smooth transition. The coefficients of the three polynomials are calculated by solving three sets of linear (in the coefficients) equations taking into account all the boundary conditions. An equal distribution of the intermediate points $\left(t_{1}\right.$ and $t_{2}$ ) makes these polynomials not oscillate unnecessarily, which can occur when using high order polynomials. In an actual implementation often some acceleration and speed conditions in the end points are neglected, lowering the order of the polynomial, which diminishes the oscillatory behavior.

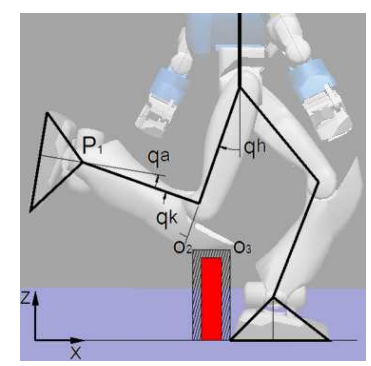

(a) intermediate point 1

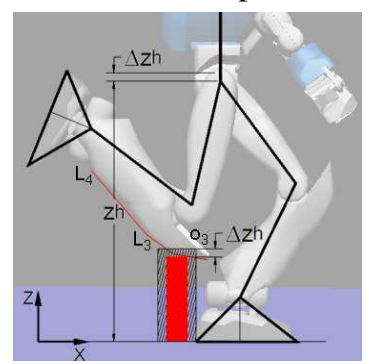

(c) height variation

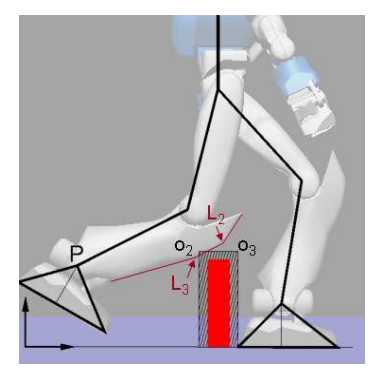

(b) collision occurs

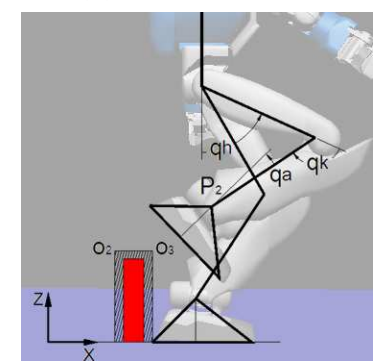

(d) intermediate point 2
Fig. 5. Several intermediate stepping over robot postures

For the preliminary study in this paper the order of the polynomials is preserved.

With the current polynomial structure on the ankle position the knee will possibly collide with the top of the obstacle during transfer of the ankle from point $P_{1}$ to $P_{2}$, especially when regarding large obstacles and the hip at the lowest chosen height of the feasibility study of the previous double support phase. During this double support phase the height of the hip was chosen low since the nature of the double support phase induces risks for overstretch, but during mid single support this is not the case anymore. And as such the hip height can be temporally increased in order to avoid collision of the knee with the obstacle. Thus, if collision occurs, an additional $6^{\text {th }}$ order correcting polynomial is constructed to induce a temporal hip height variation with a maximum height calculated as such that the knee does not interfere with the safety zone around the obstacle, as is depicted in Fig. 5c. The hip height variation $\Delta Z_{h}(t)$ is not only added to the standard hip height but also to the polynomial of the vertical ankle position:

$$
\begin{aligned}
\Delta Z_{h}(t) & =\sum_{i=0}^{6} a_{i}^{\Delta Z_{h}} t^{i} \\
Z_{h}(t) & =Z_{h_{D S}}+\Delta Z_{h}(t) \\
Z_{a}(t) & =\sum_{i=0}^{7} a_{i}^{z_{a}} t^{i}+\Delta Z_{h}(t)
\end{aligned}
$$

The polynomial function for the hip height variation takes into account smooth transition at the end points and is spread over the complete time course of the second leg stepping over phase, thus not only between points $P_{1}$ and $P_{2}$, in order not to induce too large $Z M P$ oscillations, and as such not to jeopardize the correcting performance of second preview loop. 
The collision free calculations of the intermediate points are related to absolute position of the hip, determined by the pattern generator. So, referring to the overall control scheme of Fig. 2, the information of the $C o G$ fifo buffer is required for the stepping over planner, which is provided via connection (d) in Fig. 2. This means that before the planner can establish the polynomials one preview round is performed, for which only the information of the $Z M P$, previously determined by the feasibility, is required and provided via connection $(a)$. The second preview loop needs the calculated polynomials of the stepping over planner in order to perform inverse kinematic calculations, which allows eventually to calculate the $\triangle Z M P$ buffer. After the second preview loop the $C o G$ course is adapted in order to restore dynamic stability, taking into account the effects on the dynamics by the stepping over procedure. So at this point a second stepping over planning is performed using the new positions of the $C o G$ via connection $(f)$ in Fig. 2. As such the establishment of the polynomials is repeated and the fifo buffer of foot trajectories is refreshed (connection $(c)$ in Fig. 2). This new fifo buffer is then used to perform the second inverse kinematic calculations to finally determine the exact robot states during the complete stepping over procedure.

There is still one important issue left to be tackled. The polynomial trajectory planner so far has taken into account several intermediate collision free configurations. And, although these intermediate points are selected carefully and the hip height was increased, there is no guarantee that tracking these specific ankle and hip trajectories will result in collision free stepping over, especially when large obstacles are negotiated, due to the complex movement and shape of the leg itself. So, the last required tool is an online trajectory adapter which makes small corrections to the planned base polynomial trajectories. This is the subject of the next section.

\section{Collision Free Trajectory Adapter}

Picture (b) of Fig. 5 shows that a virtual (with the safety boundary) collision occurs with the knee near point $o_{2}$. So, in order to assure a safe global stepping over, the current ankle trajectory is adapted such that the leg always stays out of the safety boundary of the obstacle. The idea is to create a discrete online trajectory adapter which starts with a newly created polynomial, fitted on the base ankle polynomial, and this piecewise on parts of the trajectories separated by the intermediate collision free control points $P_{1}$ and $P_{2}$, e.g. the polynomial trajectory between the rear double support ankle point $P_{0}$ and point $P_{1}$ (see left of Fig. 6). Two $6^{\text {th }}$ order polynomials (for $X$ and $Z$ ) are now fitted on the original trajectory, taking into account position, velocity and acceleration conditions at the boundary points with time instants $\left(t_{n_{0}}\right),\left(t_{n_{1}}\right)$ and one position condition in between. Next, at each sample $n$ (filling the fifo feet trajectory buffer), these new polynomials are redesigned analogously but over the remaining time course and with a specific point in between at time instant $t_{n_{c d}}$. At the future sample $n_{c d}$ is checked if a collision occurs, thus predicting $\left(n_{c d^{-}}-n\right)$ samples ahead. If a collision occurs

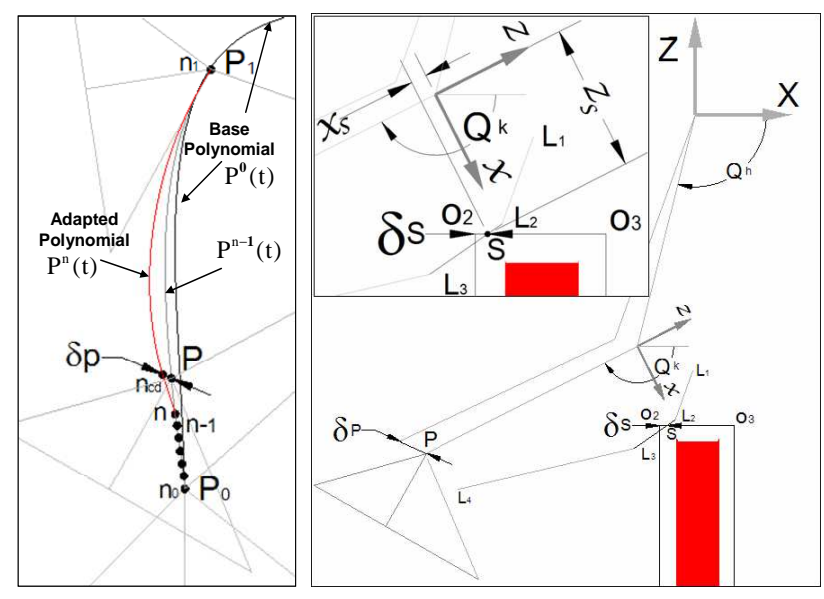

Fig. 6. Online collision free polynomial adaptation principle

at this future position $P$, a displacement $\delta p$ is applied which is required for the ankle to move in order to avoid the collision. Subsequently, the $6^{\text {th }}$ order polynomials $\left(P_{X}^{n}(t)\right.$ and $\left.P_{Z}^{n}(t)\right)$ are refitted with end conditions still those of endpoint $P_{1}$, but now with begin conditions at sample point $n$, calculated with the previous polynomial designed at sample $(n-1)$. Hereby, the new collision free position $(P+\delta p)$ is taken into account. Thus e.g. $P_{X}^{n}(t)$ is constructed with following boundary conditions:

$$
\begin{gathered}
\text { Begin: } \\
X_{a}\left(t_{n}\right)=P_{X}^{n-1}\left(t_{n}\right) \\
\dot{X}_{a}\left(t_{n}\right)=\dot{P}_{X}^{n-1}\left(t_{n}\right) \\
\ddot{X}_{a}\left(t_{n}\right)=\ddot{P}_{X}^{n-1}\left(t_{n}\right)
\end{gathered}
$$

End:

$$
\begin{aligned}
& X_{a}\left(t_{n_{1}}\right)=P_{X}^{0}\left(t_{n_{1}}\right) \\
& \dot{X}_{a}\left(t_{n_{1}}\right)=\dot{P}_{X}^{0}\left(t_{n_{1}}\right) \\
& \ddot{X}_{a}\left(t_{n_{1}}\right)=\ddot{P}_{X}^{0}\left(t_{n_{1}}\right)
\end{aligned}
$$

\section{Intermediate :}

$$
X_{a}\left(t_{n_{C D}}\right)=P_{X}^{n-1}\left(t_{n_{C D}}\right)+\delta p_{X}
$$

This strategy is applied separately for the different phases, before, after and in between the collision free points $P_{1}$ and $P_{2}$. Doing so guarantees that each adapted $6^{\text {th }}$ order polynomial is collision free at the beginning and the end, for which no specific adaptation is regarded. The adaptation process of the polynomial always takes the previous correction into account and as such it is remembered, so the next correction will be small or even zero.

The correction $\delta p$ is calculated whenever a collision is detected between the regarded line segments of the leg and the line segments of the obstacle, as explained with Eqs. (2) and (3). E.g. referring to the right scheme of Fig. 6 a 
collision occurred between line segment $\left(l_{2}, l_{3}\right)$ of the leg with line segment $\left(o_{2}, o_{3}\right)$ of the obstacle. The trajectory adaptation will move the intersection point $s$ horizontally towards the point $O_{2}$ in order to leave the safety boundary region around the obstacle, since the leg is still in front of the obstacle. If this intersection occurs while the leg is already upwards, then a vertical shift will be induced. The necessary distance $\delta s$ to shift the intersection point has to be realized by an appropriate ankle point displacement $\delta p$. Thus a link between both movements is required. Due to the continuous adaptation process of the correcting polynomial, the required displacements will be small, as such jacobian calculations, with respect to a frame $(X, Z)$ attached at the hip, can be used on the swing leg in order to related $\delta s$ and $\delta p$. If two new absolute oriented angles (ccw +$) Q_{h}$ and $Q_{k}$ for the hip and knee respectively are defined with respect to the horizontal $X$, and if $S\left(x_{s}, z_{s}\right)$ is relative to the lower leg reference frame, both ankle point $P$ and collision intersection point $S$ variations can be approximated as follows:

$$
\begin{aligned}
\left(\begin{array}{l}
\delta X_{s} \\
\delta Z_{s}
\end{array}\right) & =\left(\begin{array}{cc}
-L_{u} \sin Q_{h} & -x_{s} \cos Q_{k}+z_{s} \sin Q_{k} \\
L_{u} \cos Q_{h} & -x_{s} \sin Q_{k}-z_{s} \cos Q_{k}
\end{array}\right)\left(\begin{array}{l}
\delta Q_{h} \\
\delta Q_{k}
\end{array}\right) \\
& =J_{s}\left(\begin{array}{l}
\delta Q_{h} \\
\delta Q_{k}
\end{array}\right) \\
\left(\begin{array}{l}
\delta X_{p} \\
\delta Z_{p}
\end{array}\right) & =\left(\begin{array}{cc}
-L_{u} \sin Q_{h} & -L_{l} \sin Q_{k} \\
L_{u} \cos Q_{h} & L_{l} \cos Q_{k}
\end{array}\right)\left(\begin{array}{l}
\delta Q_{h} \\
\delta Q_{k}
\end{array}\right) \\
& =J_{p}\left(\begin{array}{l}
\delta Q_{h} \\
\delta Q_{k}
\end{array}\right)
\end{aligned}
$$

with $L_{u}$ and $L_{l}$ respective lengths of upper and lower leg. Eliminating the angle variations $\delta Q$ in Eq. (8) results in the required relation between the two displacements:

$$
\left(\begin{array}{l}
\delta p_{x} \\
\delta p_{z}
\end{array}\right)=J_{p} J_{s}^{-1}\left(\begin{array}{l}
\delta s_{x} \\
\delta s_{z}
\end{array}\right)
$$

Thus Eq. (9) is used to calculate the required $\delta p$ which is applied to the polynomial construction (condition $7 \mathrm{c}$ ) in order to adapt the base ankle trajectory of the swing foot and as such avoid any collision. The final results of this online correcting strategy is depicted in Fig. 7.

The newly determined ankle positions are stored in the fifo feet trajectory buffer (Fig. 2) and the impact on the $Z M P$ is again cancelled by horizontal hip motion deviations, calculated by the second preview loop. After this second loop the fifo buffers are recalculated with the same trajectory adapter, but it is expected that changes are minor compared to the first adaptation round, so that the dynamic impact on the $Z M P$ is less. The online trajectory adapter will be active all the time, since it is not only necessary during the preview calculations, but also to react on deviations in a realtime tracking implementation. After all, this generally involves extra control loops such as the ZMP stabilizer of HRP-2 [15], which compensates for actual ZMP errors, measured with force sensors in the feet, while walking. But for the swing leg to react collision free on this control loop, the trajectory adapter will be formulated with displacements calculated on the actual sample $(n)$ instead of the preview sample $\left(n_{C D}\right)$.

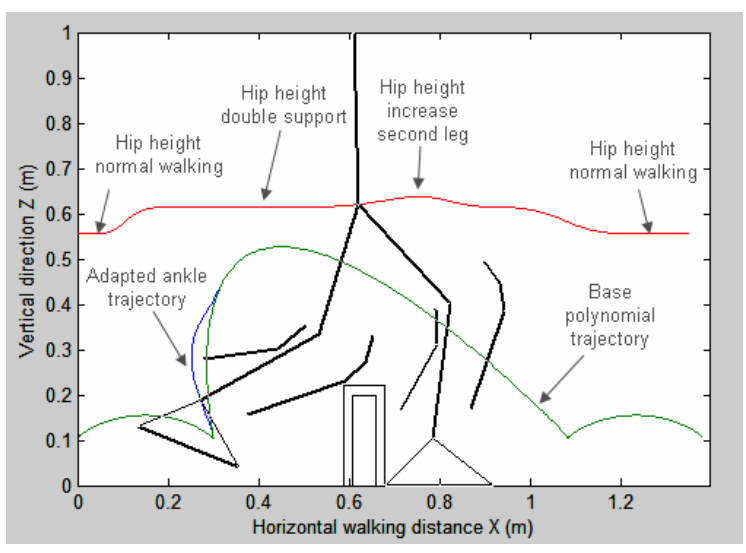

Fig. 7. Hip trajectory and adapted ankle trajectory

\section{Foot Trajectory Planner Summary}

Fig. 8 gives an overview of the foot planning strategy. At first the obstacle dimensions are given as input, which in a later stadium of the research will originate from the vision system. With the information of the dimensions and taking into account a safety boundary, the feasibility calculates a step width $X_{a_{D S}}$ and appropriate hip height $Z_{h_{D S}}$ while using an approximation of the horizontal hip position during double support in accordance with the dynamic pattern generator. Using the distance to the obstacle the footholds can be planned and the desired ZMP buffer can be completed, after choosing the different step-times. Also, starting from the step before until the step after the obstacle negotiation, the hip height is increased and decreased again with respect to the normal walking hip height and the required hip height $Z_{h_{D S}}$ during the double support of the stepping over. Subsequently, a first preview, regarding the system as a simple inverted pendulum, is performed.

Next, using the generated hip positions $X_{h}(t)$ and two chosen time instants, the collision free intermediate points $P_{1}$ and $P_{2}$ are determined after which two base polynomials for the vertical and horizontal ankle motion are established, while taking into account all conditions at begin and end of the specific leg stepping over. After applying inverse kinematics calculations, collision detections are performed to check if the leg collides with the safety boundary of the obstacle on the trajectory between points $P_{1}$ and $P_{2}$. If a collision occurs, a $\Delta Z_{h}$ is calculated, which is used to establish a polynomial to induce a temporal hip height variation during the specific single support phase.

So far the trajectory has been adapted as to minimize the risk for collision, but due to the complex movements, there is no guarantee for collision free stepping over. Especially, when large obstacles are considered and during the first phase of the second leg stepping over. Thus after the hip height increase the online trajectory adapter is applied in block B3, creating a collision free trajectory.

Finally, a second preview is performed to take into account the complete multibody model of the robot while compen- 


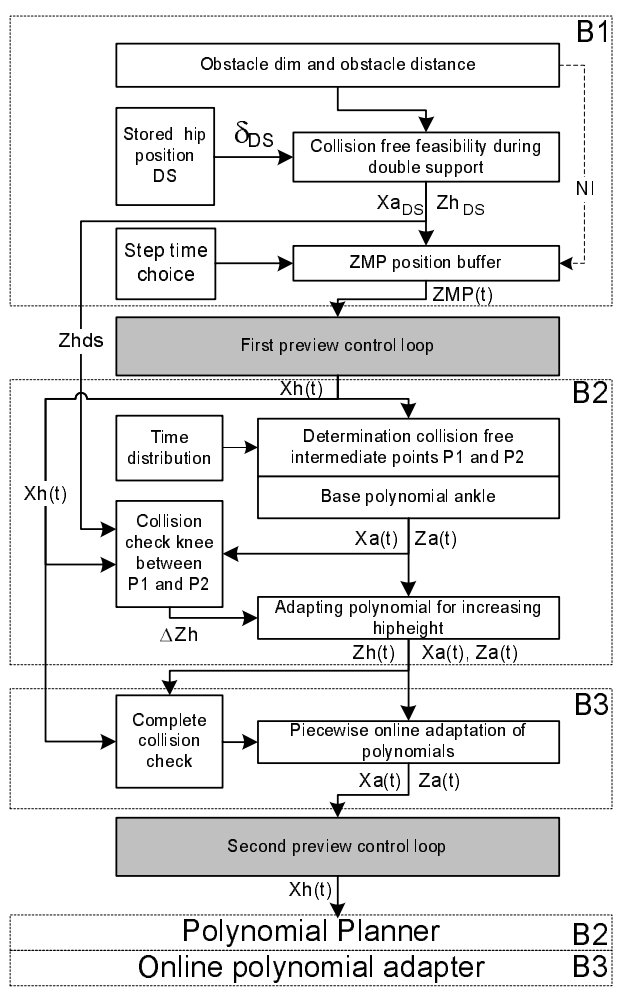

Fig. 8. Overview of the foot trajectory planner

sating the dynamical effects of large swing leg motions and the hip height increase for the stepping over procedure. The second preview loop will add correcting accelerations on the horizontal hip motion and consequently also small deviations in the hip position. Therefor the previously planned ankle trajectories might again induce an interference of some parts of the leg with the safety boundary around the obstacle. So the polynomial planning unit $B_{2}$ (Fig. 8) and online trajectory adapter $B_{3}$ are performed again to make small corrections to the ankle trajectory, hereby assuming that these small corrections will not influence the actual ZMP course too much anymore and as such not jeopardize the overall stability during the stepping over.

\section{CONClusion}

Humanoid robots have the ability to navigate through complex environments due to their legged configuration. This feature also allows such robots to negotiate obstacles by stepping over them. This paper presents a study on stepping over large obstacles dynamically, in order to perform a smooth fluent motion. The designed strategies are intended for the humanoid research platform HRP-2, and make use of the elaborate dynamic pattern generator currently implemented for regular walking in this specific biped. The preliminary study presented in this paper focusses on the sagittal plane and the dynamic implementation of large swing leg retractions and hip height variations have been discussed by means of a simplified model of the robot. A polynomial foot trajectory planner, including an online collision free trajectory adapter, has been proposed and the paper discusses in detail the interaction of this foot planner with the dynamic walking pattern generation scheme. Currently, this planning method is being extended to three dimensions and an incorporation in the real robot is planned for the near future. The final goal is to achieve a vision guided stepping over tool which can be incorporated in a path planner in order to increase the robot's mobility.

\section{ACKNOWLEDGMENT}

This research was supported by a Post-doctoral Fellowship of Japan Society for Promotion of Science (JSPS).

\section{REFERENCES}

[1] K. Hirai, M. Hirose, Y. Haikawa, and T. Takenaka, "The development of honda humanoid robot," in Proc. IEEE International Conference on Robotics and Automation, (Leuven, Belgium), pp. 1321-1326, 1998.

[2] S. Thompson and S. Kagami, "Humanoid robot localisation using stereo vision," in Proc. IEEE International Conference on Humanoid Robots, (Tsukuba, Japan), pp. 19-25, IEEE, 2005.

[3] A. Davidson, O. Stasse, and K. Yokoi, "Vision-based SLAM for a humanoid robot," in Proc. IEEE International Conference on Robotics and Automation (Workshop), (Barcelona, Spain), IEEE, April 2005.

[4] C. Burghart, R. Mikut, R. Stiefelhagen, T. Asfour, H. Holzapfel, P. Steinhaus, and R. Dillmann, "A cognitive architecture for a humanoid robot: A first approach," in Proc. IEEE International Conference on Humanoid Robots, (Tsukuba, Japan), pp. 357-362, IEEE, December 2005.

[5] N. Ee Sian, K. Yokoi, S. Kajita, and K. Tanie, "A stable foot teleoperation method for humanoid robots," in Proc. IEEE International Conference on Robotics and Automation, (New Orleans, USA), pp. 1065 - 1070, April 2004.

[6] M. Gienger, H. Janß en, and C. Goerick, "Task-oriented whole body motion for humanoid robots," in Proc. IEEE International Conference on Humanoid Robots, (Tsukuba, Japan), pp. 238-244, December 2005.

[7] K. Sabe, M. Fukuchi, J.-S. Gutmann, T. Ohashi, K. Kawamoto, and T. Yoshigahara, "Obstacle avoidance and path planning for humanoid robots using stereo vision," in Proc. IEEE International Conference on Robotics and Automation, (New Orleans, USA), pp. 592-597, April 2004.

[8] P. Michel, J. Chestnutt, J. Kuffner, and T. Kanade, "Vision-guided humanoid footstep planning for dynamic environments," in Proc. IEEE International Conference on Humanoid Robots, (Tsukuba, Japan), pp. 1318, December 2005.

[9] K. Kaneko, F. Kanehiro, S. Kajita, H. Hirukawa, T. Kawasaki, M. Hirata, K. Akachi, and T. Isozumi, "Humanoid robot HRP-2," in Proceedings of the IEEE International Conference on Robotics and Automation, (New Orleans, USA), pp. 1083-1090, 2004

[10] Y. Guan, K. Yokoi, E. Neo, and K. Tanie, "Feasibility of humanoid robots stepping over obstacles," in Proc. Of Int. Conf. On Intelligent Robots and Systems (IROS), (Sendai, Japan), pp. 130 - 135, IEEE, September 2004.

[11] Y. Guan, K. Yokoi, and K. Tanie, "Feasibility: Can humanoid robots overcome given obstacles?," in Proc. IEEE International Conference on Robotics and Automation, (Barcelona, Spain), pp. 1066-1071, IEEE, April 2005.

[12] M. Vukobratovic and B. Borovac, "Zero-moment point - thirthy five years of its life," International Journal of Humanoid Robotics, vol. 1, pp. 157-173, 2004.

[13] S. Kajita, F. Kanehiro, K. Kaneko, K. Yokoi, and H. Hirukawa, "The 3d linear inverted pendulum mode: A simple modelling for a biped walking pattern generation.", in Proceedings of the IEEE International Conference on Intelligent Robots and Systems, (Maui, Hawai), pp. 239-246, 2001.

[14] S. Kajita, K. Kanehiro, K. Kaneko, K. Fujiwara, K. Harada, K. Yokoi, and H. Hirukawa, "Biped walking pattern generation by using preview control of zero-moment point," in Proc. IEEE International Conference on Robotics and Automation, (Taipei, Taiwan), pp. 1620-1626, IEEE, 2003.

[15] K. Yokoi, F. Kanehiro, K. Kaneko, K. Fujiwara, S. Kajita, and H. Hirukawa, "A honda humanoid robot controlled by AIST software," in Proceedings of the IEEE/RAS International Conference on Humanoid Robots, (Tokyo, Japan), pp. 259-264, 2001 